Nutr. et Dieta 1959;1:I-IV

\title{
Contents, Vol. 1, 1959
}

\section{Nutritio et Dieta}

Europäische Zeitschrift für Ernährung und Diätetik European Review of Nutrition and Dietetics Revue européenne de nutrition et de diététique

EDITORES

E. J. BIGWOOD, B $\pi$ ixelles $H$.

H. H. BERG, Hamburg

E.

R. BOLLER, Wien J.

E. LE BRETON, Paris F.

L. BRULL, Liège A.

F. S. P. VAN BUCHEM, Groningen H.

M. COPPO, Modena A.

H. D. CREMER, Giessen R.

CH. DARNAUD, Toulouse G.

J. DELFOSSE, Bruxelles $\mathrm{H}$.

M. DEMOLE, Geneve T.

M. J. L. DOLS, Wassenaar G.

G. DUCHESNE, Paris A.

H. GLATZEL, Dortmund H.

W. GLOOR-MEYER, Zurich J.

J. GONTEA, BucarestF.

H. GOTJNELLE, Paris L.

W. HALDEN, Graz G.

C. DEN HARTOG, Den Haag G.

R. JACQUOT, Paris F.

B. C. P. JAN SEN, Amsterdam R.

KRAUT, Dortmund

I. KÜHNAU, Hamburg

LEDERER, Louvain

MANCINI, Roma

P. MEIKLEJOHN, Edinburgh

G. MOGENA, Madrid

MOSSE, Paris

NICOLAYSEN, Oslo

PEQUIGNOT, Paris

SARLES, Marseille

F. S. M. VAN SCHAIK, Den'Haag

S. SCHETTLER, Stuttgart

VAN DER SCHUEREN, Gand

M. SINCLAIR, Oxford 
C. SOMOGYI, Zurich

J. TRAISSAC, Bordeaux

TRAVIA, Roma

P. VECCHI, Modena

VERDONK, Gand

VERZAR, Basel

WENGER, Wien

REDACTORES

E. AZERAD, Paris J. TREMOLIERES, Paris

SECRETARIUS H. KAPP, Basel

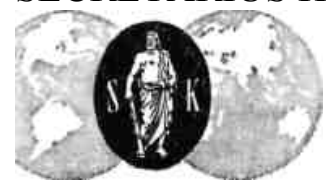

1959

Vol. 1

BASEL (Schweiz)

S. KARGER

NEW YORK

INDEX

Übersichtsreferate - Surveys - Revues générales

Azerad, E.: L'action des acides gras non-saturés sur le taux du cholesterol sanguin. - Die

Wirkung der ungesättigten Fettsäuren aufden Cholesteríngehalt des Blutes. - The Action of Unsaturated Fatty Acids on the Level of Blood Cholesterol 73

Berg, D.: Ernährung und degenerative Gefäßkrankheiten. - Nutrition and Degenerative

Diseases of the Blood Vessels. - Alimentation et affections dégénératives des vaisseaux 133

Bourde, Ch. et Milliat, A.: Diététique des affections veineuses des membres. - Díätetík

der Venenkrankheiten. - Dietetics of the Venous Affections of the Limbs 193

Milliat, A.: vide Bourde, Ch.

TrémoliÈres, J.: Vues actuelles sur Tutilisation des standards nutritionnels pour juger une ration alimentaire. - Moderne Beurteílung der Standardwerte der Ernährung. - Present-day Views on the Utilisation of Nutritional Standards (of Measure) in the Evaluation of an Alimentary Ration 4

Origínalarbeiten - Original Paper - Travaux orígínaux

Abraham, J.: vide Jacquot, R.

Babinet, J. P., Veniard, M. et Heraud, G.: Variation de la glycémie au cours de Гeffort musculaire intensif Suppl. 17

Bernier, J.J.: vide TrémoliËres, J.

Bigwood, E. J.: vide Nunnikhoven, R.

Bosley, E. M.: vide Kincses, A.

Brand, V. von ; Drescher, H. und Zöllner, N.: Über die Verwertung parenteral zugeführter Fettemulsionen und ihre Beeinflussung durch Heparin. - On the Utilization of the Administration of Parenteral Fat Emulsions and the Effect Produced by Heparin. - Sur l'utilisation d'émulsions huileuses administrées par voie parentérale et ses modifications sous l'influence de Théparine 161

Brunaud, M.: vide Jacquot, R. 
Darnaud, C.; Denard, Y.; Moreau, G. et Voisin, R.: Les effets de la cortisone sur la stéatose nutritionnelle expérimentale du foie. - Wirkung des Cortísons aufdie experimentelle nahrungsbedíngte Fettleber. - The Effect of Cortisone on Experimental Nutritional Steatosis of the Liver 237

De Langen, C. D.: Nutritional Factors other than Fats and Lipoids which Influence the Development of Atherosclerosis. - Beeinflussung der Entwicklung der Atherosklerose durch Nichtfettstoffe. - Influence des facteurs nutritionnels autres que les graisses et les lipoïdes sur le développement de Гathérosclérose 227

Delfosse, J.: Alimentation de collectivités en cas de sinistre Suppl. 52

Demole, M.: vide Kincses, A.

Denard, Y.: vide Darnaud, C.

Desaga, H.: Nouvelles Recherches sur le pain et son emploi dans la diététique de Tartériosclérose et du diabète Suppl. 157

Dobersky, P.: Le système diététique dans les centres balnéaires tchécoslovaques Suppl. 91

Drescher, H.: vide Brand, V. von

Dvorsky, A.: L'alimentation et la dépense d'énergie des kayakistes pendant l'entraînement d'hiver pour le XXVe Championnat du monde de kayak à Prague . . . Suppl. 25

Dvorsky, A.: L'alimentation collective en Slovaquie Suppl. 100

Fornoza, A.: Glucose, vitamine Bx et amino-acides en sport Suppl. 2

Gounelle, H. et Leclerc, M.: La teneur en vitamine $\mathrm{C}$ du legume en plat cuisine. - Der

C-Vítamíngehalt des tíschfertigen Gemüses. - The Level of Vitamin C Content in Cooked Vegetables 121

Guarienti, F.: vide Vecchi, G.P.

Heraud, G.: vide Babinet, J. P.

Horky, J.: Einige Probleme der Gemeinschaftsverpflegung von Seeleuten bei Schiffsfernfahrten Suppl. 105

Horky, J.: Ein Beitrag zur Frage der Eiweißernährung bei einigen chronischen Erkrankungen Suppl. 197

Jacquot, R.; Abraham, J.; Petrovic, Y.; Segal, V.; Brunaud, M. et Trémolières, J.: Efficacité alimentaire et dépense calorique d'entretien du rat suivant la nature des graisses du regime. Abhängígkeít der Nahrungsverwertung und des kalorischen Erhaltungs-umsatzes der Ratte von der Art der Nahrungsfette. - Alimentary Efficiency and Calory Expenditure for the Maintenance of the Rat According to the Nature of Fats in the Diet 214

Jacquot, R.; Abraham, Y.; Raveux, R.; Brunaud, M. et Trémolières, J.: Mobilisation des graisses de reserve suivant la nature des graisses alimentaires chez le rat. - Abhängígkeit der Mobilisation der Fettreserven bei der Ratte von der Art der Nahrungsfette. - Mobili sation of Fat Storage to the Nature of the Alimentary Fats in the Rat 221

Kahr, E.: Die Ernährung stationärer Krebskranker. - The Alimentation of Stationary Cancer Patients. - L'alimentation des cancéreux hospitalises 126

Kincses, A.; Demole, M. et Bosley, E. M.: La place du regime hyperprotéiné dans la thérapeutique des cirrhoses hépatiques Suppl. 168

Le Bideau, G. et Rivolier, J.: Le travail en haute altitude. Ses implications nutritionnelles et diététiques Suppl. 28

Leclerc, M.: vide Gounelle, $\mathrm{H}$. 
Lederer, J. et Stein, F.: Le regime hyperprotéiné dans les gangrenes diabëtiques - Role adjuvant du phényl-propionate de nor-androstanolone Suppl. 182

Lowy, R.: vide Trémolières, $\mathrm{J}$.

Milliat, A. L. et Milliat, F. M.: Alimentation et sport automobile .... Suppl. 43

Milliat, F. M.: vide Milliat, A. L.

Mitrovic, M.: Diététique et vitaminothérapie dans la Pellagre. - Díätetík und Vitamíntherapíe der Pellagra. - Dietetics and Vitamintherapy in Pellagra 150

Moreau, A.: vide Verdonk, G.

Moreau, G.: vide Darnaud, C.

Nunnikhofen, R. et Bigwood, E. J.: Variation de la composition en acides amines de la farine de froment en fonction du pourcentage de son extraction du grain de blé. - Variatíonen des Amínosäurengehaltes des Weízenmehls in Abhängígkeít vom Ausmahlungsgrad. Variation in the Composition of Amino Acids in Wheat Flour as a Function of the Per centage Extraction from Corn Grain .177

Österreicher, I.: vide Wenger, R.

Petrovic, Y.: vide Jacquot, R.

Raveux, R.: vide Jacquot, R.

Rilliet, B.: Le traitement du diabétique artérioscléreux. Le role de la diététique dans la prevention et la thérapeutique des complications vasculaires propres au

diabète Suppl. 112

R $\gamma$ volieb, J.: vide Le B $\pi>$ eau, G.

Rubbiani,. V.: vide Vecchi, G.P.

Saetti, G.C.: vide Vecchi, G.P.

Segal, V.: vide Jacquot, R.

Stein, F.: vide Lederer, J.

Trémolières, J.; Bernyer, J. J. et Lowy, R.: Etudes sur les modalités de digestion de divers types d'amidon. - Untersuchungen über die Verdauungsvorgänge bei verschiedenen Stârkearten. - A

Study of the Modalities of Digestion of Different Types of Starches . . 100

Tréholyëres, J.: vide Jacquot, R.

Vander Schueren, A.: vide Verdonk, G.

Vangelabbeek, H.: vide Verdonk, G.

Vangelabbeek, H.: La situation alimentaire de la region minière limbourgeoise. - Die

Ernährungslage im Bergwerksgebiet von Limburg. - The Alimentary Situation in the

Mining District of the Province of Limburg 90

Vecchi, G. P.; Rubbiani, V.; Saetti, G. C. and Guarienti, F.: Dietary Consumption in

Patients with Myocardial Infarction. (Notes on a Nutritional Investigation). - Der

Nahrungsmittelverbrauch von Patienten mit Myokardínfarkt. (Bemerkungen über die Erhebung der Ernährungslage). - La consommation diététique chez des patients avec infarctus du myocarde. (Notes sur une enquête alimentaire) 27

Veniard, M.: vide Babinet, J. P.

Verdonk, G.: Diététique de l'athérosclérose. Rapport general Suppl. 124

Verdonk, G.; Vander Schueren, A.; Moreau, A. et Vangelabbeek, H.: Etude de

Talimentation dans les collectivités: sanatoriums, homes d'enfants, homes de vieillards, prisons et hôpitaux en Belgique Suppl. 64 
Verdonk, G.: Experiences diétêtiques dans la clinique de Гathéromatose. - Dietätische Versuche in der Klinik der Atheromatose. - Dietetic Experiments in the Clinical Approach of Atheromatosis 41

Voisin, R.: vide Darnaud, C.

Wenger, R. und Österreicher, I.: Mais- und Sonnenblumenöl in der Diätetik. - Maize and Sunflower Oil in Dietetics. - L'huile de maïs et de tournesol en diététique 66 Zöllner, N.: vide Brand, V. von.

GeselL·chaftsberichte - Society Transactions - Sociétés

5"* Journées Européennes de Diététique, Bruxelles/Gand/Louvain, du 26 au 29 septembre 1959 Suppl.

Congrès International de Gastro-Entêrologie et de Nutrition, Karlovy-Vary (Tchécoslovaquie) 21-24 septembre 1959248

Referate - Abstracts - Analyses $\quad 71,191,251$

Buchbesprechungen - Book Reviews - Livres nouveaux 130

Varia 72,132

Vorwort - Foreword - Avant-propos 1

Alle Reehte, insbesondere das der Überßetzung in fremde Sprachen, vorbehalten.

Ohne ausdrückliche Genehmigung des Verlages ist es auch nicht gestattet, diesen Band oder

Teile

derails anf photomechanischem Wege (Photokopie, Mikiokopie) zu vervielfältigen.

Copyright 1959 by S. Karger AG, Basel

Printed in Switzerland

Cliches: Aberegg-Steiner \& Cie., AG, Bern und Steiner \& Cíe., AG, Basel

Druck: Lüdin AG, Liestal 